\title{
Exploring Political Ad Libraries for Online Advertising Transparency: Lessons from Germany and the 2019 European Elections
}

\author{
Juan Carlos Medina Serrano \\ juan.medina@tum.de \\ Technical University of Munich \\ Munich
}

\author{
Orestis Papakyriakopoulos \\ Technical University of Munich \\ Munich, Bavaria \\ orestis.papakyriakopoulos@tum.de
}

\author{
Simon Hegelich \\ Technical University of Munich \\ Munich, Bavaria \\ simon.hegelich@hfp.tum.de
}

\begin{abstract}
This study investigates the possibilities and limits presented by the newly created ad libraries from Facebook and Google to analyze online political campaigns. We selected Germany as a case study and focused on the months leading up to the 2019 elections to the European Parliament. We identified the political actors that were active advertisers, compared their spending, and contrasted the number of ad impressions with user engagement on their organic online content. From the political ads, we extracted the unique ads and manually analyzed a subsample of them. Furthermore, we explored regional and demographic distributions of users reached by the advertisements and used them as a proxy for the advertisers' targeting strategies. We also compared the success of the ad campaigns on boosted Facebook posts. We found that even though all the major German political parties engaged in online ad campaigns, they kept their attempts at microtargeting to a minimum. Although their Facebook-sponsored posts were more successful than normal posts, we did not find statistical significance for all the political parties. Interestingly, we noticed that the distribution of users reached by the right-wing party Alternative für Deutschland (AfD) diverges from that of the other parties. Finally, we discuss further challenges for enhancing transparency in online advertising.
\end{abstract}

\section{CCS CONCEPTS}

- Information systems $\rightarrow$ Display advertising; Social networks; - Human-centered computing $\rightarrow$ Social network analysis.

\section{KEYWORDS}

political campaigns, ad libraries, social media, European elections, Facebook, Google, transparency

ACM Reference Format:

Juan Carlos Medina Serrano, Orestis Papakyriakopoulos, and Simon Hegelich. 2020. Exploring Political Ad Libraries for Online Advertising Transparency: Lessons from Germany and the 2019 European Elections. In International Conference on Social Media and Society (SMSociety '20), Fuly 22-24, 2020,

Permission to make digital or hard copies of all or part of this work for personal or classroom use is granted without fee provided that copies are not made or distributed for profit or commercial advantage and that copies bear this notice and the full citation on the first page. Copyrights for components of this work owned by others than ACM must be honored. Abstracting with credit is permitted. To copy otherwise, or republish, to post on servers or to redistribute to lists, requires prior specific permission and/or a fee. Request permissions from permissions@acm.org.

SMSociety '20, July 22-24, 2020, Toronto, ON, Canada

(C) 2020 Association for Computing Machinery.

ACM ISBN 978-1-4503-7688-4/20/07 . \$ \$15.00

https://doi.org/10.1145/3400806.3400820
Toronto, ON, Canada. ACM, New York, NY, USA, 11 pages. https://doi.org/ $10.1145 / 3400806.3400820$

\section{INTRODUCTION}

The creation of ad libraries represents the largest effort to date to introduce transparency in online advertising. Ad libraries are collections of online ads with corresponding information on who funded them, how much was spent, and general information on the users that saw them. Ad libraries can be supported with application programming interfaces (APIs), which provide a direct channel to extract information. Before ad libraries existed, online political ads were classified as dark ads as they were publicly inaccessible [44]. This enabled astute malicious actors to target susceptible groups. Online political manipulation was not thoroughly considered in the early years of the online platforms. However, there was a turning point after the 2016 US presidential election with the Cambridge Analytica scandal. The company was charged with sending customized ad messages based on psychographic profiles of US citizens using private data from Facebook [72]. Further reports on data misuse and misinformation campaigns on Facebook and other online platforms followed. This included divisive and polarizing Facebook ads from the Russian Internet Research Agency, which were intended to incite social conflict and polarize society in the US [62]. In response to these scandals and escalating international pressure, Facebook decided to increase transparency in online advertisement. The social media company created an ad archive report in the weeks before the 2018 US midterm elections and announced the first ad library API. Facebook made the ad library public in March 2019. Google and Twitter followed and launched their ad transparency libraries a few months before the 2019 European elections.

The elections for the European Parliament were held between May 23 and 26, 2019. They constituted the ninth elections since the first parliamentary elections in 1979. Twenty-eight countries participated in the process of selecting 751 Members of the European Parliament. For this study, we focused on Germany, the member state with the largest population. The case of Germany is of interest since 1) the country has strong data privacy laws [23]; 2) Facebook, Google, Instagram, and YouTube are still the primary channels for online political ads [21], in contrast to other countries where political actors also use messaging apps [61]; and 3) Germany has a far-right populist party that behaves differently than the other German parties [63].

The purpose of this paper is to show the extent to which Facebook's and Google's ad libraries can help in understanding online campaigns. For this purpose, we quantify the online advertising 
campaigns of the major German political parties in the months leading up to the 2019 European elections. We first compare political party spending and the differences between ad impressions and user interactions on organic content. Organic content refers to the content that was shared on Facebook pages or YouTube channels but was not promoted by ad campaigns. Second, we explore the number of unique ads per political party and give a general overview of the content of the ad. We then try to understand political parties' targeting strategies using the demographic and regional distributions of users reached by the advertisements. Finally, we quantify the ad campaigns' success by comparing user engagement between sponsored posts and organic posts on Facebook.

Studies by Edelson et al. [24] and Ghosh et al. [33] presented a first analysis of the ad libraries. They analyzed the different types of political advertisers in the US in 2018 and aimed to understand the targeting features that the advertisers used. However, neither study focused on the political parties, the content of the ads, or user interactions. Moreover, the studies explored the libraries when they were still in beta testing. This paper takes a further step toward the establishment of methodologies to analyze online political advertising with the help of the ad libraries in combination with additional data sources. This makes it possible to obtain a detailed overview of online political campaigns during election periods.

\section{BACKGROUND}

\subsection{Online Advertising}

With the widespread use of digital services, online advertisement is becoming increasingly ubiquitous. Worldwide spending on digital marketing is predicted to reach $\$ 333$ billion in 2019, which accounts for roughly half of the global ad market [25]. There are numerous types of online advertising such as search advertising, social media advertising, and email advertising [35]. For all types of advertising, the business model is based on continuous data collection of personal information, monitoring of individuals' online behavioral patterns, and pursuit of customer manipulation. This new form of business model is at the core of what has been referred to as the "digital surveillance economy" [19]. Its main innovation relies on targeting users at an unprecedented granular level. Microtargeting is defined as the creation of customized messages delivered to groups or individuals that are predicted to be impacted by those messages [1]. It relies on the full gamut of big data algorithms to define differentiable and profitable customer groups [58]. Apart from targeting, online advertisement allows for increased measurability, since every user's response to ads can be easily tracked [35].

Social media platforms and search engines create user profiles by collecting as much information as possible on their users' online activity [9] from their interactions, such as likes, dislikes, comments, and connections, $[6,17]$ to their browsing behaviors via cookies [46]. With the collected data, advertisers are provided with fine-grained targeting features, including personally-identifiable information (PII), such as phone numbers and email addresses [71]. Facebook allows targeting of users' behavior, location, interests, demographics, and connections [26], and Google allows targeting of their website visits, interests, locations, and demographics [37]. After advertisers select a target audience, a matching mechanism decides which ads to deliver to a given user. There is a real-time auction to display an ad to a user, where the ad of the higher bidder is chosen [32].

A controversial category of online advertisement is political advertisement, given the possible influence of political ads on public opinion. In the last few years, political campaigns have increasingly embraced data-driven targeting strategies [39, 48, 57]. Online political advertisement as the main vehicle of these targeting strategies has reshaped modern-day politics [18]. Political actors are now able to identify and reach individual users who are more likely to be persuaded by them and to match their messages to the users' specific interests and vulnerabilities [74]. Narrower targeting allows politicians to tailor advertising on wedge issues, such as immigration and abortion, to sway pivotal voters [40]. A data-driven online campaign makes microtargeting a powerful technique and is currently the state of the art in political campaigning. In the US, political parties have made this possible by amassing a huge amount of personal data on voters' political affiliations and behavior [10]. Despite having the strictest data privacy regulations, Germany is also a fertile ground for political microtargeting [59]. Apart from political parties, other advertisers also activate ads with political intent. It is, however, not a straightforward task to define the borders of what constitutes a political ad. Facebook and Google have implemented different policies to decide which ads are added to the ad libraries. Facebook's archive includes all advertisements that have political content, are regulated as political advertising or are related to a national legislative issue. On the other hand, Google only includes advertisements either created by political actors or related to specific elections [24].

Few studies have examined the breadth of political parties' online advertisement, largely because of the unavailability of relevant datasets. However, there is extensive social science literature on political advertising-from studies that focus on analyzing the content of political ad campaigns $[5,66,70]$ to research experiments that attempt to determine if political advertising is successful in persuading voters $[31,34,51]$. These studies agree that political advertising has the potential to persuade, but its real impact depends on the user characteristics. In this way, political advertising can both influence users' vote choice and prompt mobilization on election day [54]. Facebook conducted a randomized controlled trial of political mobilization messages delivered to 61 million users, which resulted in an increased turnout [14]. It is possible that the political advertising on social media platforms can motivate similar effects.

\subsection{Data Privacy and Transparency}

With the rise of online advertisement and microtargeting, serious concerns have been raised about data privacy and transparency $[30,46,69,74]$. Prior work has uncovered the use of sensitive data for Facebook advertisement targeting purposes [16]. For example, Venkatadri et al. [71] noted the possibility of targeting individuals with sensitive PII, such as phone numbers provided for security purposes and phone numbers derived from friends' contact lists. Furthermore, attackers can exploit the vulnerabilities of the advertising interfaces to breach user privacy [47] or to employ discriminatory targeting [22, 65]. On the subject of transparency, Kreiss and Mcgregor [49] consider that both Facebook and Google have 
been opaque in their decision-making, following policies that are not transparent and that were applied without explicit justification.

Facebook and Google have taken steps to address the aforementioned concerns. Both platforms have introduced an ads preference page where users can see and correct the information that was inferred about them $[29,36]$. They have also introduced information explaining why each ad was displayed. However, Andreou et al. [3] found the explanations on Facebook to be incomplete and sometimes misleading. Additionally, Datta et al. [22] reported incomplete representations of user profiles on Google's ad settings. Apart from these design flaws, it is essential to consider the user perspective. One concern is that users may not be aware of the advertising transparency mechanisms. A 2019 Pew Report [41] found out that $74 \%$ of Facebook users in the US did not know that the platform maintains a list of their interests and traits to target them. A second concern is that users may not care about transparency in the usage of their private data. The well-studied privacy paradox suggests that even though users are concerned about privacy online, their behaviors do not reflect these concerns [7]. For instance, Baum et al. [8] found that the presence of political ads on mobile apps does not consequentially deter users from choosing such an app. In the case of Germany, the findings of Taddicken et al. [68] indicate that privacy concerns hardly have an effect on users' self-disclosure.

\subsection{Ad Effectiveness}

The field of ad effectiveness is explored extensively in the literature as it constitutes the main goal of a successful marketing campaign. Kingsnorth [45] presents three types of measurement metrics in online advertisement: traffic metrics, such as impressions and clickthrough rate (number of clicks/impressions); conversion metrics, in which a conversion refers to the number of users who clicked on the ad and then successfully triggered a specific action such as buying a product or subscribing to a newsletter; and efficiency metrics, with the most common one being the return on investment (ROI), which is the revenue or profitability divided by the ad cost. These measures are connected to business value when the advertisement is directly linked to a physical product or a service. However, this is not the case when the ad's purpose is to raise customer awareness, such as promoting a brand or a political candidate [42]. Calculating a "political" ROI is challenging given that it is hard to find effective and standardized metrics for measuring the impact of online political advertising. Previous political campaigns in the US have used as metrics the number of dollars fundraised after a user clicked on a search ad, and the number of ad volunteer sign-ups gathered as a result of ad click-throughs [20]. However, this information is only available to the campaigns, which does not allow a comparison between different political parties. A useful set of metrics relies on user engagement. User engagement is defined as the quality of the user experience that emphasizes the positive aspects of the interaction and in particular what motivates a user to interact with a web application [52]. User engagement metrics differ from each online platform depending on their design. They range from the number of unique users, click-through rates, page views, and time spent on a website. A subclass of user engagement refers to online behavior metrics [52]. For social media, these include direct user interactions with the advertising posts, such as likes, comments, and shares. These metrics have already been used as a measure of success in literature. Poecze et al. [60] analyzed social media metrics to evaluate the effectiveness of YouTube videos. Lee et al. [50] and Jaakonmäki et al. [43] studied the relationship between ad content and user engagement to measure the effectiveness of social media content marketing campaigns on Facebook and Instagram, respectively. Additionally, previous research has found a positive relationship between user interactions and election results [11, 38]. This suggests that user interactions could also be a proxy for ad effectiveness. Unfortunately, there is no clear methodology to measure how online behaviors translate into offline actions. Research has found a strong connection between online user engagement and behavioral intentions, and simultaneously between the expression of intention and message-induced offline behaviors [2, 73]. Unsurprisingly, the online media platform's algorithms are programmed to optimize user engagement [15]. User engagement allows the collection of vast amounts of data. This contrast to the relatively scarce data on voting behavior as elections are held once or twice every two years. Nevertheless, it remains a challenge to correctly quantify ad effectiveness. With the extensive datafication of politics, new metrics could appear in the near future.

\section{METHODOLOGY}

\subsection{Data Collection}

We collected data from political ads in Germany using the newly created Facebook Ad Library API and the Google Cloud BigQuery API. The former includes ads shown both on Facebook and Instagram, whereas the latter has ads that appeared on Google Search and YouTube. We employed a daily procedure to collect data from both APIs to avoid missing ads that could be later removed. Moreover, this allowed us to circumvent the technical difficulties and bugs that appeared after a few days during the collection dates [55] and collect all the ads that targeted Germany. The collection period we selected began with the creation day for each API (beginning and middle of March 2019, for Facebook and Google respectively), and ended one week after the European elections. In total, the dataset comprises 50,794 Facebook ads and 34,197 Google ads. The content of the ads is often not unique since ad campaigns constantly deploy similar ads, which either contain a small modification, have a different target strategy, or are distributed on different platforms. However, each ad has a unique ID, which allows us to avoid duplicates in the daily collection process.

We also gathered the content and interactions from the Facebook posts and YouTube videos published by the main political parties in Germany. These are the Christian Democratic Union (CDU), the main center-right party; the Christian Social Union (CSU), the sister party of the CDU operating only in the state of Bavaria; the AfD, the far-right party; Bündnis/Die Grünen, the green party; the Free Democratic Party (FDP), a neoliberal centrist party; the Social Democratic Party of Germany (SPD); and Die Linke, the left party. For each party, we further obtained the data for the regional pages and channels from the 16 German states.

To collect data from Facebook and YouTube, we used CrowdTangle and the YouTube Data API, respectively. The collection period corresponds to the same dates we selected for the ads. The dataset 
consists of 11,496 Facebook posts and 1,059 YouTube videos. Unfortunately, we were not able to collect data from Instagram, given that most of the capabilities from its public API are deprecated. In addition, we exclude Twitter ads as we did not find political ads from national or regional German party accounts on the Twitter Transparency Center.

\subsection{Content Analysis}

The first step in our analysis was to manually match the advertisers on Facebook and Google to political parties. Each political party has a main advertiser and other smaller advertisers connected to accounts of German states, cities, and local politicians. We placed the advertisers that did not belong to a political party into the following categories: government; companies and organizations, which include NGOs and labor unions; and others.

Our analysis mainly focuses on the advertisement created by the main and regional advertisers for the aforementioned German political parties. From the ads created by these advertisers, we filtered out duplicate ads. This was not a trivial task since the APIs do not provide a direct way to compare images or videos. Identical images that belong to different ads are stored as copies in the ad archives and can only be compared visually. For this reason, we crawled the ad libraries with the help of Selenium and downloaded the images. It was not necessary to download the videos as the unique URL link (for Google, a link to a YouTube video) was contained in the HTML of the website.

To find unique advertisements, we used string matching between texts and the Perceptual Hashing algorithm [53] to compare images. This algorithm creates a unique hash for an image. Hashes can be then compared using the Hamming distance. Since advertisers often use the same image in different sizes or pixel qualities, the hashes are not completely identical. After several tries, we considered images similar if the Hamming distance between the respective hashes was smaller than 20. After identifying the list of unique ads, we analyzed a random sample of 30 ads per party and per platform to give us a general overview of the advertising content and party intentions. Our focus was to evaluate whether the ad content was personalized or not. Defining personalization is not straightforward as it has a complete taxonomy [12]. For us, personalization is correlated to the content breadth of messages spread by a political party. An ad campaign with similar messages and scarce content would be classified as not personalized.

\subsection{Demographic and Regional Strategies}

The Facebook advertisement data contains the demographic and regional distribution of the users reached by each advertisement. These distributions depend on the targeting distribution that the advertisers selected but are not the same. They also depend on Facebook's algorithm and the users who are active at a given time. However, since we do not have access to the specific targeting strategies, we take the distributions of reached users as proxy variables for the original targeting strategies. We consider this limitation in the discussion section.

The demographic distribution is divided according to gender (including unknown) and six age ranges, whereas the regional distribution corresponds to the 16 German states. We first averaged the regional and demographic distributions for all ads belonging to a given party. We then computed the correlation between each pair of political parties. We subsequently averaged the correlations for each party separately. This procedure resulted in a number between -1 and 1 that conveys the similarity between a party's strategy and the remaining parties. The higher the score, the more similar are the targeting distributions. A low score denotes dissimilarities to the rest of the parties or in the case of a negative value, opposite strategies. We excluded the CSU for the regional distribution as it is only represented in one of the German states.

Additionally, we compared the regional distribution with the electoral support in each state. We obtained the percentage of voter intention by opinion polls conducted between February and May $2019^{1}$. For each party, we divided the percentage of approval for the German states by the sum of the percentages to normalize the data and obtain a variable from 0 to 1 that represents state popularity. We then calculated the correlation between state popularity and the mean regional distribution. A positive correlation would indicate that a party targeted states with high voter support. On the other hand, a negative correlation would relate to a strategy targeting states with lower voter support. We also excluded the CSU from this analysis.

\subsection{Campaign Performance}

We seek to quantify the success of the German parties' ad campaigns. The success of an ad campaign can be measured through the performance of the ads, either by click-rate or by the extent of user engagement with the ad. We use the number of total interactions to measure success as the click-rate information is only available to the advertisers. It is impossible to quantify real success, in other words, if the users were politically persuaded, but analyzing user engagement is a common practice in marketing to estimate success.

The core idea of the method is to investigate whether increased sponsorships have a significant effect on the number of user interactions. The level of sponsorship can be quantified with the number of impressions as these two quantities correlate. However, they are not completely equivalent given that advertisers can decide to pay for either clicks or impressions. According to Facebook documentation [27], impressions correspond to the number of times the ads were on-screen for the target audience. This means that if an ad is shown twice to the same user, it counts as two impressions. Similarly, Facebook interactions are not unique since one user can comment, share, and choose one of six reactions (like, wow, angry, haha, love, and sad) for a given post.

Facebook divides advertisements into boosted posts and regular ads [28]. Boosted posts are already existing posts on a public Facebook page, whereas regular ads are created separately from scratch. To test performance, we focus only on boosted posts since it is possible to compare user engagement between sponsored and non-sponsored posts. The Facebook Ad Library API neither differentiates between the ad type nor provides the number of interactions per ad. To obtain this information, we matched the ad data with the collected posts from the Facebook pages. We used the text description and the image or video of the ads and posts to automatically match between them. Only the ads belonging to boosted posts had

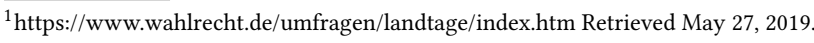


a direct match. In total, the seven German parties boosted 522 posts from their national and regional Facebook pages.

One limitation to the ads data is that the exact number of impressions is unknown. For each ad, the API only reports a lower and an upper bound. These bounds belong to predefined ranges by Facebook. Only the advertiser who launched the ads can access the real values for impressions. We use the middle value between the lower and upper bound as an approximation to the real value. Additionally, each post can be sponsored by more than one ad. Therefore, we define total mean interaction as the sum of the middle values of the ads that sponsor a given post.

To compare between sponsored and non-sponsored posts while considering the level of sponsorship, we divide the posts into four groups; not sponsored, low-level sponsor, middle-level sponsor, and high-level sponsor. We define the level boundaries by grouping the 522 sponsored posts into three even groups ordered according to the ads' total mean interaction.

The distributions of social media interactions are generally nonnormal, and most of them follow a log-normal distribution [4]. By plotting the distribution of the total mean interactions, we confirm that the non-normality holds. Consequently, we perform KruskalWallis tests, which are the non-parametric version of the one-way ANOVA. We then apply post-hoc pairwise Wilcox tests with Bonferroni corrections to compare between the non-sponsored group and each of the sponsored groups. Given that the dependent variable has intrinsic errors that we are not able to control, we set a stricter significance level of $\mathrm{p}<0.01$.

One limitation of this approach is that it neither controls for the time of posting nor for post content. A complete controlled experiment would compare posts with similar content that were launched at the same point in time. However, the purpose of our analysis is only to test the general performance of the parties' ad campaigns and obtain an indicator of overall success.

Although a similar analysis on Google ads can be performed on sponsored YouTube videos, the dataset contains only 23 videos. The rest of the ads correspond exclusively to ad videos, or to another ad category on Google, such as text or image. We present the KruskalWallis test results of the 23 videos combined, without distinguishing between parties. For YouTube, the number of total interactions consists of views, comments, likes, and dislikes from videos posted by the political parties.

\section{RESULTS}

\section{Ad Campaigns}

The German political parties implemented ad campaigns on the platforms belonging to Facebook and Google. Table 1 shows the number of ads and the spending in Euros for the parties per platform. For Facebook, we differentiate between the federal and state advertisers, which we refer to as main advertisers, and the local advertisers, which correspond to cities and individual politicians. From the main advertisers, the CDU spent more money and activated more ads than the other parties. The SPD comes in a close second place. However, when the local advertisers are included, the SPD comes in first place. On the other hand, the CSU, AfD and Die Linke were the parties that allocated less money on ads from both Facebook categories and Google advertisers. The CSU is only active in the state of Bavaria and had no Google ads during the observed period. Although the period for the Google ads is two weeks shorter than the Facebook ads period, it is evident that most of the parties spent more money on the latter.

Table 1: Facebook ads and spending in the period from March 1 to June 2, 2019 and Google ads from March 20 to June 2, 2019. The Facebook ads are divided into two categories: by country-wide and regional political pages, and city and individual political pages. Facebook political pages that spent less than 100 Euros are not included.

\begin{tabular}{l|rr|rr|rr|}
\hline & \multicolumn{2}{|c|}{ Facebook } & \multicolumn{2}{c|}{ Facebook (Local) } & \multicolumn{2}{c|}{ Google } \\
& Ads Spending & Ads & Spending & Ads Spending \\
\hline AfD & 48 & 22,278 & 255 & 9,029 & 17 & 23,400 \\
CDU & 17,449 & 296,801 & 1,755 & 53,141 & 33,120 & 261,200 \\
CSU & 27 & 60,816 & 66 & 6,611 & 0 & 0 \\
FDP & 5,456 & 138,762 & 970 & 60,691 & 259 & 32,600 \\
Die Grünen & 7,804 & 229,451 & 1,888 & 60,283 & 769 & 140,750 \\
Die Linke & 958 & 41,526 & 359 & 7,259 & 7 & 3,200 \\
SPD & 15,234 & 283,664 & 3,174 & 130,692 & 90 & 133,900 \\
\hline
\end{tabular}

The Facebook local advertisers spent less money than the main advertisers for the seven parties. However, the amount spent by the local advertisers is still significant. We do not show the local advertisers on Google as we only found three city advertisers and each spent less than 2,000 Euros in the investigated period. The results that follow concentrate only on the main advertisers per party.

\section{User Interactions}

The party spending translates into ad impressions, and then the impressions into clicks. Table 2 presents the upper and lower bound of total impressions from all the ads belonging to a given party. We opted to show the one percent of the bounds since click-through rates on Facebook are in this order of magnitude [56]. This is a rough approximation of users interacting with the ads, and the values' order of magnitude is more significant than the exact number. The impressions from Facebook include Instagram, and those from Google include YouTube. For comparison, we include the number of total interactions on Facebook posts and YouTube videos uploaded in the same period. These correspond to organic interactions on the parties' social media channels. For the AfD, Die Grünen and Die Linke, organic content exceeds the one percent upper bound of the number of impressions. This is not the case for the FDP and the SPD. Nevertheless, these interactions do not take into account the missing Instagram and Google search data. A comparison between the political parties reveals that the AfD has more interactions than the other German parties.

\section{Unique Ads}

The perception of the number of ads created by the political parties changes when we take into consideration only the unique ads. The German political parties created little advertising content as 
Table 2: Lower and upper bound of impressions generated by ads. The $1 \%$ represents the order of magnitude of click-through rates. For Facebook, the interactions correspond to likes, shares, comments, and reactions. For YouTube, the interactions consist of views, comments, likes, and dislikes.

\begin{tabular}{|c|c|c|c|c|c|c|}
\hline & \multicolumn{2}{|c|}{ Facebook/Instagram Impressions } & \multirow{2}{*}{$\begin{array}{r}\text { Facebook } \\
\text { Interactions }\end{array}$} & \multicolumn{2}{|c|}{ Google/YouTube Impressions } & \multirow{2}{*}{$\begin{array}{r}\text { YouTube } \\
\text { Interactions }\end{array}$} \\
\hline & Lower (1\%) & Upper (1\%) & & Lower (1\%) & Upper (1\%) & \\
\hline$A f D$ & 17,600 & 39,019 & $2,632,650$ & 27,600 & 276,200 & $5,674,531$ \\
\hline$C D U$ & 143,780 & 639,673 & 256,493 & 148,600 & $4,659,300$ & $3,412,524$ \\
\hline$C S U$ & 23,750 & 55,109 & 197,422 & 0 & 0 & 42,041 \\
\hline$F D P$ & 169,850 & 576,462 & 268,756 & 74,200 & 751,200 & 260,839 \\
\hline Grünen & 141,900 & 474,408 & 262,415 & 49,900 & 559,200 & $2,387,547$ \\
\hline Linke & 59,050 & 158,404 & 253,981 & 11,100 & 111,400 & 348,821 \\
\hline$S P D$ & 177,540 & 638,163 & 245,865 & 32,900 & 332,100 & 288,682 \\
\hline
\end{tabular}

illustrated in Table 3. The table is divided by type of advertising. For Facebook, there are images, videos and events, and for Google there are images, YouTube videos and ads with only text that appear on Google searches. Even though the CDU activated by far the largest number of ads, its unique number of ads is distinctively small, for example, only six Google images and three YouTube videos. The FDP, Die Grünen and the SPD created more content for their campaigns. These results show that the political parties were mostly activating the same ads repeatedly during the months leading up to the 2019 European elections.

Table 3: The number of unique ads and the total number of ads per political party. The ads are divided by their type and platform. The number of unique ads is presented in bold. For Facebook, some ads were not included in the three categories, were not found in the Ad Archive anymore, or were duplicate ads from the advertisers. For this reason, the exact number may differ from Table 1.

\begin{tabular}{l|rrr|rrr|}
\hline & \multicolumn{3}{|c|}{ Facebook } & \multicolumn{3}{c|}{ Google } \\
& Image & Video & Event & Image & Video & Text \\
\hline$A f D$ & $\mathbf{1 9} / 22$ & $\mathbf{1 7} / 17$ & $\mathbf{2} / 2$ & $\mathbf{1} / 1$ & $\mathbf{6} / 7$ & $\mathbf{3} / 9$ \\
$C D U$ & $\mathbf{3 4 / 3 1 1}$ & $\mathbf{2 4} / 12,316$ & $\mathbf{4} / 7$ & $\mathbf{6} / 28,139$ & $\mathbf{3} / 4,981$ & $/ 0$ \\
$C S U$ & $\mathbf{1 2} / 17$ & $7 / 10$ & $/ 0$ & $/ 0$ & $/ 0$ & $/ 0$ \\
$F D P$ & $\mathbf{3 9 4} / 3,991$ & $\mathbf{6 8} / 722$ & $\mathbf{3 3} / 58$ & $\mathbf{2 7} / 178$ & $\mathbf{1 2} / 80$ & $/ 0$ \\
Die Grünen & $\mathbf{1 3 4} / 1,606$ & $\mathbf{7 8} / 2,448$ & $\mathbf{3 5} / 53$ & $\mathbf{3} / 33$ & $\mathbf{9} / 63$ & $\mathbf{2 1 5} / 609$ \\
Die Linke & $\mathbf{6 7 / 1 1 1}$ & $\mathbf{4 1} / 234$ & $\mathbf{5 4} / 167$ & $\mathbf{1} / 1$ & $\mathbf{2} / 2$ & $\mathbf{4} / 4$ \\
SPD & $\mathbf{2 2 3 / 7 , 7 4 8}$ & $\mathbf{6 6} / 3,826$ & $\mathbf{3 4} / 75$ & $\mathbf{1} / 1$ & $\mathbf{1 1} / 11$ & $\mathbf{5 4} / 78$ \\
\hline
\end{tabular}

After inspecting a random batch of 30 ads per party and per platform, we found that many unique ads are similar to each other. The parties did not diversify their content and adopted similar content and format for their advertising. As an example, Figure 1 shows one representative ad for three of the political parties. From the ads we observed, we noticed that the CDU created fewer personalized messages than the other parties. Even though the AfD activated few ads, their advertising was more personalized by using long texts with several URLs included. The FDP, Die Grünen and the SPD concentrated on their main campaign proposals, and they presented a few ideas outside of the topics that distinguish these political parties. Interestingly, the FDP created several ads in languages other than German, for example, Russian and Greek, to try to reach different parts of the electorate.

\section{User Distributions and Polls}

Figure 2 compares the regional and demographic distributions of users reached by the targeting parties. We compare the parties by providing a mean correlation value. The SPD has the highest value for the regional distribution, which means it is the most similar to the other parties. By contrast, the AfD has a correlation value close to zero, which corresponds to the party with the most dissimilar regional pattern. The difference is even larger for the demographic distribution, where the AfD's correlation value is negative. This represents a completely opposite demographic pattern to the audience who perceived the AfD's ads. The SPD also has a lower mean correlation value than the other parties, whereas the FDP has the highest value.

The left image from Figure 2 corresponds to the correlation between opinion poll values and the mean regional distribution. We observe that on average the AfD, SPD and Linke targeted and reached users in states with lower voter support. On the other hand, the CDU, Die Grünen and FDP targeted and reached on average the states where voter support was higher. The average correlation value shows a general tendency for the political parties. This tendency is hard to observe by only analyzing the individual regional distributions per ad as all parties targeted repeatedly the 16 German states.

\section{Effectiveness of Sponsored Posts}

For the Facebook posts, we compare the relationship between impressions and the number of total interactions as a proxy of an ad campaign's success. For each political party, Table 4 shows the ratio between the mean value of total interactions from sponsored posts and the mean value from non-sponsored posts. The FDP has 

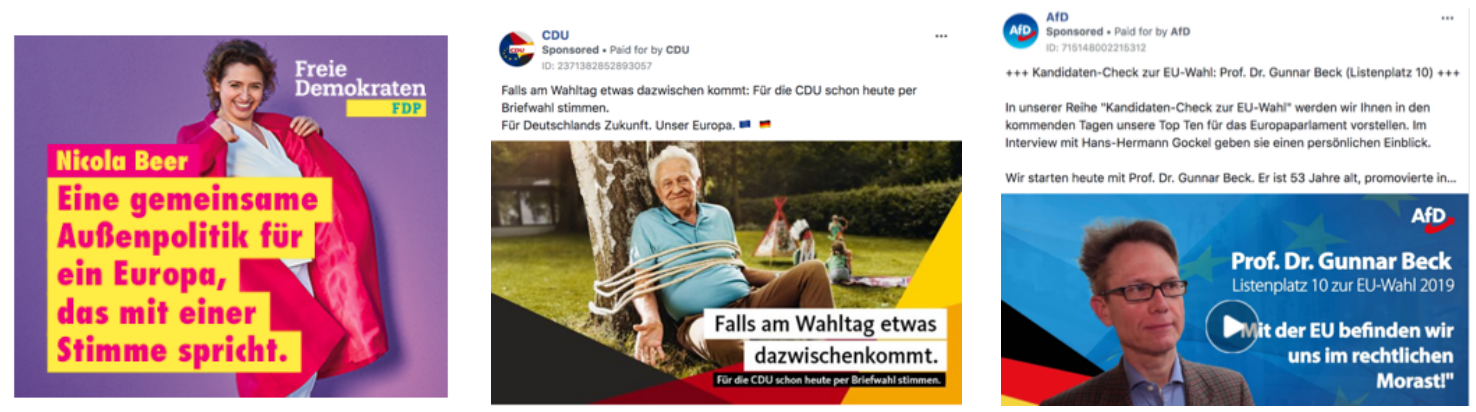

Figure 1: Example of three ads during the campaign. Right: FDP image on Google. Middle: CDU image on Facebook. Left: AfD video on Facebook.
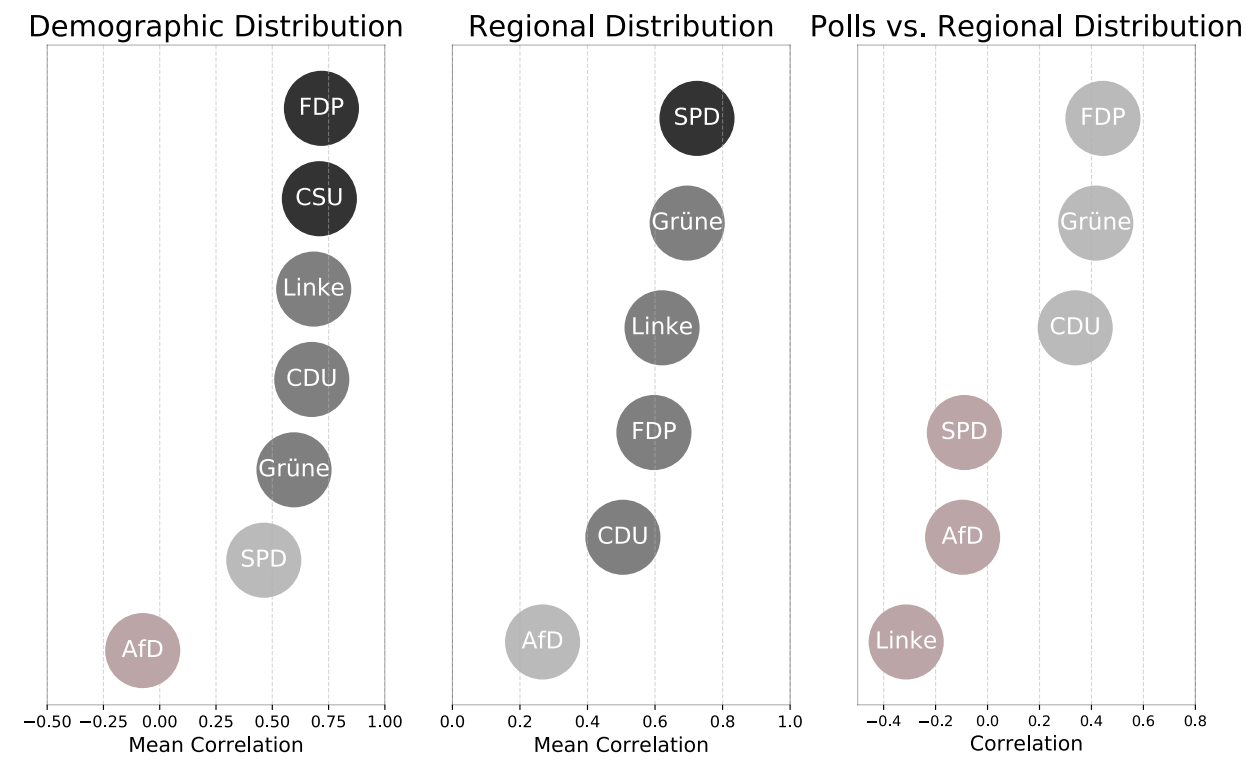

Figure 2: Left and Center: Demographic and regional distributions of users reached by Facebook ads. The mean correlation value corresponds to overall distribution similarity. It is an average value of correlations between the ad distributions for one party and each of the rest. Right: Correlation between voter support from opinion polls and mean regional distribution.

the biggest ratio value, whereas the CDU and CSU have the lowest values. On average, the sponsored posts from all the political parties had more interactions than the non-sponsored posts. However, this metric is not enough to show that there was a significant improvement given that it does not take into account the distributions of the interactions on the posts. Table 4 further presents the Kruskal-Wallis tests between four groups of posts: non-sponsored, low-sponsor, middle-sponsor and high-sponsor. The table also reports the results from the post-hoc pairwise Wilcoxon tests between the non-sponsored posts and each of the sponsored groups. For the CDU and CSU, there are no significant differences between groups, which is further confirmed by the three pairwise Wilcoxon tests. Even though there is significance in the group differences for Die Grünen and Die Linke, the pairwise tests reveal that the difference is only significant for highly sponsored ads. Finally, for the AfD, FDP, and SPD, the Kruskal-Wallis tests are significant as are the post-hoc tests between the non-sponsored posts and the other three groups. In the case of YouTube, we perform the statistical tests on the videos of the parties together because of the limited dataset of sponsored videos. We find a significant difference between the groups of videos $(\mathrm{p}<.001)$.

\section{Ad Categories}

As a final analysis on the Facebook ad archive, we present the comparison between advertisers belonging to different categories. Given that Facebook considers advertisements related to both political issues and topics, there are other advertisers apart from the political parties in the archive. Table 5 shows the percentage of ads and total spending from the advertisers of the four categories. We observe that although $86 \%$ of the ads were created by the political parties, only $50 \%$ of the total spending can be credited to them. Private companies, NGOs, and labor unions represent $25 \%$ of the total ad spending on the social media platform. Further analysis of 
Table 4: The ratio between the means of total interactions from sponsored posts and non-sponsored posts. Additionally, statistical tests between the interactions of nonsponsored posts and sponsored posts divided in low, middle and high level of sponsorship. The p-value corresponds to the Kruskal-Wallis tests for the difference between the groups. In parenthesis, the post-hoc pairwise Wilcoxon tests with Bonferroni correction between non-sponsored posts and each of the other three groups (low, middle, high). Significance codes: ${ }^{* * *} \mathrm{p}<0.001,{ }^{* *} \mathrm{p}<.005,{ }^{*} \mathrm{p}<0.01, \bullet \mathrm{p}>0.01$

\begin{tabular}{lrrr}
\hline & $\begin{array}{r}\text { Ratio of } \\
\text { means }\end{array}$ & K-W tests & $\begin{array}{r}\text { Pairwise W tests } \\
\text { (low, middle, high) }\end{array}$ \\
\hline$A f D$ & 2.45 & $\mathrm{p}<.001$ & $\left(\begin{array}{c}* * * * * * *) \\
(\bullet, \bullet, \bullet)\end{array}\right.$ \\
$C D U$ & 1.52 & $\mathrm{p}=.17$ & $(\bullet, \bullet, \bullet)$ \\
$C S U$ & 1.88 & $\mathrm{p}=.039$ & $($ *********) \\
FDP & 4.23 & $\mathrm{p}<.001$ & $\left(\bullet, \bullet,,^{* * *}\right)$ \\
Die Grünen & 2.19 & $\mathrm{p}<.001$ & $(\bullet, \bullet,, * *)$ \\
Die Linke & 2.84 & $\mathrm{p}<.001$ & $(* * * * * * * *)$ \\
SPD & 3.49 & $\mathrm{p}<.001$ & \\
\hline
\end{tabular}

Table 5: Percentage of number of ads and spending from the advertising on Facebook. Advertisers are divided into four categories.

\begin{tabular}{lrr}
\hline Category & Ads (\%) & Spending (\%) \\
\hline political parties & 86.17 & 48.23 \\
government & 0.41 & 20.81 \\
companies/organizations & 10.68 & 25.72 \\
other & 2.73 & 5.23 \\
\hline
\end{tabular}

political ads from other advertisers than political parties is outside the scope of this paper.

\section{DISCUSSION}

For the first time, it is possible to scrutinize the digital marketing campaigns of political parties. This opens up the possibility of holding political actors accountable for their online marketing spending and the messages they promote. The provided APIs helped us in obtaining the data through a direct and automatic process that allowed us to frequently update our database. However, APIs could present technical challenges for some users. During the studied period, we observed the technical difficulties and bugs that have been previously discussed [55]. Nevertheless, we collected data several times a day to have the most complete dataset possible. From the information perspective, we find two main limitations in the ad libraries. First, the spending and impressions per ad are only available in broad ranges with different sizes. This reduces the statistical models that can be implemented using the data. A better technique would be for the companies to provide the original quantities with some random noise added. In this way, well-known models, such as linear regression would present more accurate results. A second limitation is that there is no information on the original user-targeting strategies from the advertisers. We understand that such information would reveal campaign strategies to competitors, but this shortcoming limits the transparency potential of online political advertising.

Our findings in the case of Germany show that the major political parties were actively using the online advertisement platforms. In the three months leading up to the 2019 European elections, the CDU had the biggest ad campaign, with the most money spent and the largest number of ads. The SPD and Die Grünen also had very active campaigns on both platforms. Between the 2017 German federal election and the 2019 European elections, these two parties saw their electoral results change considerably. The SPD's acceptance slipped from $20.5 \%$ to $15.6 \%$, whereas Die Grünen's approval increased from $8 \%$ to $20 \%$ [67]. We avoid comparing election results with ad campaigns as it is impossible to quantify a political conversion rate.

The possible impact of the online political campaigns for the 2019 European elections reduces when we consider the low number of unique ads created by the German political parties. We conclude that few attempts were made at implementing microtargeting strategies as this technique requires personalized messages that target different parts of the electorate. However, it remains possible that the correct users were targeted and have to some extent been mobilized to vote. However, it is unfeasible to calculate the success rate of online advertising and user voting. From the content of the ads, we observe that the political parties in Germany mostly advertised general messages to the targeted users. It is possible that since the European elections are not designed to tackle domestic issues, the political parties did not focus on diversifying their online advertising. Manually inspecting a random sample of advertising gave us a general impression of each party's strategy. A thorough qualitative analysis of the advertising would require the examination of theoretical frameworks designed for understanding political advertising.

For all the parties, user engagement is higher on average on the promoted Facebook posts. However, for the CDU and CSU, the statistical tests do not allow us to conclude that a significant difference exists. This could indicate a general lack of user interest in interacting with their content or that the boosted posts were less attractive than the other posts. If we take the ad content into consideration, the explanation could be related to the lack of personalized ads created by the two parties. By contrast, even low-sponsored posts showed a significant increase in interactions for the AfD, SPD, and FDP. These parties' advertising content was also more diverse and personalized. It is important to emphasize that this analysis only focuses on boosted posts and excludes the remaining Facebook ads. Moreover, the real success can only be measured by comparing click-through rates between the political parties' ad campaigns, which are not publicly available data. We want to emphasize that we are not linking ad effectiveness to approval ratings, as political advertising may have a weak indirect effect on political campaigns. We only focus on the success of a political marketing campaign without implying anything in the effects on real user persuasion. Another limitation in our analysis is the missing original targeting 
strategies. The comparison between distributions of users reached by Facebook ads can be interpreted as a proxy of the real targeting by the parties. However, this could be inaccurate given that the bias of Facebook's algorithm and other external factors cannot be quantified.

Throughout this study, the AfD's results deviate from the rest. The AfD advertisers did not spend a substantial amount of money on ads and launched few ads. They mostly relied on organic channels to spread their message. The interactions on the AfD's Facebook pages and YouTube channels are accordingly the highest among the political parties. This is consistent with research on the AfD's success on social media [63]. Additionally, the AfD's demographic and regional distributions on Facebook differ from those of the other parties. This could suggest that the AfD is targeting a separate group of the population. This disjunction could be considered as a proxy for the polarization of German society. Indeed, Shahrezaye et al. [64] reported that on Facebook the polarization between the AfD and the other political parties has increased in the last few years.

Overall, this study has presented the new opportunities and limitations of the ad libraries in order to explore the online advertising. They represent the first iteration of valuable tools necessary to achieve complete transparency. Under the current scenario, we present four challenges that remain to be addressed.

The first challenge relates to the definition of a political ad. Until now, no EU regulations state what constitutes a political ad on digital channels. Without a clear formalization, the responsibility of flagging political ads resides solely with private companies. As mentioned previously, Google only includes political actors in its ad archive, in contrast to Facebook's political content policy for selecting political ads. Hypothetically, if the non-political advertisers on Facebook would have activated political ads through Google's platforms in the same proportion as they did on Facebook, over $10 \%$ of ads and $45 \%$ of spending would be unaccounted for in Google's ad library.

Given the large number of ads on both platforms, the companies most probably employ automatic classification mechanisms in the selection of political ads. The accuracy of these algorithms is not presented in their transparency reports. A second challenge is that the classification system's vulnerabilities can be exploited by malicious actors. They would try to stay undetected by sponsoring ads that may appear to have non-political content but that are deliberately intended to influence and even polarize public opinion.

The third challenge is that the increase in transparency can make political actors feel subject to scrutiny. This can, in turn, lead them to change their digital political strategies. For example, they could focus on private communication channels like WhatsApp. The endto-end encryption allows the spread of any form of political content to stay undetected. Although WhatsApp is still an unexplored channel for German political actors, this is not the same in other parts of the world, as in the case of the misinformation scandals in the 2018 Brazilian election [13].

The final challenge is that whereas online platform companies have responded to the backlash of election manipulation, government legislation has not kept up with the pace. As a consequence, the companies can make the first proposals and thus set the rules that define the problems. In such a scenario, the government will have to adapt to the proposed rules instead of leading the efforts that foster more transparency and privacy. Further steps need to be taken by both private and government institutions to deal with these challenges. Private institutions should provide services that enhance transparency and respect user privacy, whereas governmental institutions should focus on creating new regulations and raising public awareness.

\section{CONCLUSION}

In this paper, we explored methods to quantify the data from Facebook's and Google's newly created ad libraries and help further understand online political campaigns. We chose to examine the advertising from political parties in Germany in the months leading up to the 2019 European elections. We focused on spending, impressions, and the possible targeting strategies of the major German parties. We concluded that during the months leading up to the election, the parties engaged in few microtargeting attempts as they created few unique ads, with many of them lacking personalized messages. In this study, we also compared the ads' data with the political parties' organic content on Facebook and YouTube. For a selected group of ads, we introduced a way to measure success even without the ads' click-through rates. The boosted posts on Facebook attracted more user engagement than organic posts, although we did not find significant differences for all German political parties. Our findings also show that the distribution of users reached by the AfD's advertising deviated from that of the other parties, which could imply a targeting strategy that was contrary to other political parties' strategies. Furthermore, the far-right party relied mostly on promoting their organic content and not so much on sponsored ads. Finally, we introduced the challenges that arise with the transparency of ad libraries. We hope that this work helps future scholars to explore the ad libraries in different countries to understand the global political online advertising environment. A lack of continuous analysis and auditing undermines the full potential of transparency tools. It is essential to undertake further research on how to profit from these tools to enhance transparency and prevent malicious activity on online advertising platforms.

\section{REFERENCES}

[1] Tom Agan. 2007. Silent marketing: Micro-targeting. Penn, Schoen and Berland Associates (2007).

[2] Saleem Alhabash, Juan Mundel, and Syed Ali Hussain. 2017. Social Media Advertising: Unraveling the Mystery Box. In Digital Advertising. Routledge, 285-299.

[3] Athanasios Andreou, Giridhari Venkatadri, Oana Goga, Krishna P. Gummadi, Patrick Loiseau, and Alan Mislove. 2018. Investigating Ad Transparency Mechanisms in Social Media: A Case Study of Facebooks Explanations. In NDSS. The Internet Society.

[4] Sitaram Asur, Bernardo A. Huberman, Gábor Szabó, and Chunyan Wang. 2011. Trends in Social Media: Persistence and Decay. In ICWSM. The AAAI Press.

[5] Andrew O Ballard, D Sunshine Hillygus, and Tobias Konitzer. 2016. Campaigning online: Web display ads in the 2012 presidential campaign. PS: Political Science \& Politics 49, 3 (2016), 414-419.

[6] Oana Barbu. 2014. Advertising, microtargeting and social media. Procedia-Social and Behavioral Sciences 163 (2014), 44-49.

[7] Susan B. Barnes. 2006. A privacy paradox: Social networking in the United States. First Monday 11, 9 (2006).

[8] Katharina Baum, Stefan Meißner, Olga Abramova, and Hanna Krasnova. 2019. Do they Really Care about Targeted Political Ads? Investigation of User Privacy Concerns and Preferences. In ECIS. AIS.

[9] Anja Bechmann. 2013. Internet profiling: The economy of data intraoperability on Facebook and Google. MedieKultur: fournal of media and communication research 29, 55 (2013), 72-91. 
[10] Colin J Bennett. 2016. Voter databases, micro-targeting, and data protection law: Can political parties campaign in Europe as they do in North America? International Data Privacy Law 6, 4 (2016), 261-275.

[11] Adam Bermingham and Alan Smeaton. 2011. On using Twitter to monitor political sentiment and predict election results. In Proceedings of the Workshop on Sentiment Analysis where AI meets Psychology (SAAIP 2011). Asian Federation of Natural Language Processing.

[12] Jan Blom. 2000. Personalization: A Taxonomy. In CHI '00 Extended Abstracts on Human Factors in Computing Systems. ACM, 313-314.

[13] Anthony Boadle. 2018. Facebook's WhatsApp flooded with fake news in Brazil election. Reuters. Retrieved September 06, 2019 from https://www.reuters.com/article/us-brazil-election-whatsappexplainer/facebooks-whatsapp-flooded-with-fake-news-in-brazil-electionidUSKCN1MUOUP.

[14] Robert M Bond, Christopher J Fariss, Jason J Jones, Adam DI Kramer, Cameron Marlow, Jaime E Settle, and James H Fowler. 2012. A 61-million-person experiment in social influence and political mobilization. Nature 489, 7415 (2012) 295-298.

[15] C-SPAN. 2019. Technology Companies and Algorithms. Video. Retrieved September 06, 2019 from https://www.c-span.org/video/?462071-1/technologycompanies-algorithms.

[16] José González Cabañas, Ángel Cuevas, and Rubén Cuevas. 2018. Unveiling and Quantifying Facebook Exploitation of Sensitive Personal Data for Advertising Purposes. In USENIX Security Symposium. USENIX Association, 479-495.

[17] John Cheney-Lippold. 2018. We are data: Algorithms and the making of our digital selves. NYU Press.

[18] Jeff Chester and Kathryn C Montgomery. 2017. The role of digital marketing in political campaigns. Internet Policy Review 6, 4 (2017).

[19] Roger Clarke. 2019. Risks inherent in the digital surveillance economy: a research agenda. Journal of Information Technology 34, 1 (2019), 59-80.

[20] Michael Cornfield and Kate Kaye. 2009. Online Political Advertising. In Politicking online: The transformation of election campaign communications, Costas Panagopoulos (Ed.). Rutgers University Press, 163-175.

[21] Jeannette Cwienk. 2017. Wahlkampf auf Facebook, Twitter und Co. DW (2017). Retrieved January 20, 2020 from https://www.dw.com/de/wahlkampf-auffacebook-twitter-und-co/a-37535854.

[22] Amit Datta, Michael Carl Tschantz, and Anupam Datta. 2015. Automated experiments on ad privacy settings. Proceedings on privacy enhancing technologies 2015, 1 (2015), 92-112.

[23] Eco. 2017. Germany: Land of Data Protection and Security - but why? dotmagazine (2017). Retrieved January 20, 2020 from https://www.dotmagazine.online/issues/security/germany-land-of-dataprotection-and-security-but-why.

[24] Laura Edelson, Shikhar Sakhuja, Ratan Dey, and Damon McCoy. 2019. An Analysis of United States Online Political Advertising Transparency. arXiv preprint arXiv:1902.04385 (2019).

[25] Jasmine Enberg. 2019. Digital Ad Spending 2019 Global. eMarketer (2019) https://www.emarketer.com/content/global-digital-ad-spending-2019.

[26] Facebook. 2019. Find your audience. Retrieved September 06, 2019 from https://www.facebook.com/business/ads/ad-targeting.

[27] Facebook. 2019. Impressions. Retrieved September 06, 2019 from https://www.facebook.com/business/ help/675615482516035.

[28] Facebook. 2019. The Difference Between Boosted Posts and Facebook Ads. Retrieved September 06, 2019 from https://www.facebook.com/business/ help/317083072148603.

[29] Facebook. 2019. Your ad preferences. Retrieved September 06, 2019 from https://www.facebook.com/ads/preferences/.

[30] Casey Fiesler and Blake Hallinan. 2018. "We Are the Product": Public Reactions to Online Data Sharing and Privacy Controversies in the Media. In Proceedings of the 2018 CHI Conference on Human Factors in Computing Systems (CHI '18). ACM, Article 53, 13 pages.

[31] Michael M Franz and Travis N Ridout. 2007. Does political advertising persuade? Political Behavior 29, 4 (2007), 465-491.

[32] Aritz Arrate Galán, José González Cabañas, Ángel Cuevas, María Calderón, and Rubén Cuevas Rumin. 2019. Large-Scale Analysis of User Exposure to Online Advertising on Facebook. IEEE Access 7 (2019), 11959-11971.

[33] Avijit Ghosh, Giridhari Venkatadri, and Alan Mislove. 2019. Analyzing Political Advertisers' Use of Facebook's Targeting Features. IEEE Symposium on Security and Privacy. Workshop on Technology and Consumer Protection (2019).

[34] Guy J Golan, Stephen A Banning, and Lisa Lundy. 2008. Likelihood to vote candidate choice, and the third-person effect: Behavioral implications of political advertising in the 2004 presidential election. American Behavioral Scientist 52, 2 (2008), 278-290.

[35] Avi Goldfarb and Catherine E Tucker. 2011. Privacy regulation and online advertising. Management science 57, 1 (2011), 57-71.

[36] Google. 2019. Ad Settings. Retrieved September 06, 2019 from https://adssettings.google.com/.
[37] Google. 2019. Audience targeting. Retrieved September 06, 2019 from https://support.google.com/adwords/edi tor/answer/1052569?hl=en\&ref_topic $=2996408$.

[38] Brian Heredia, Joseph Prusa, and Taghi Khoshgoftaar. 2017. Exploring the effectiveness of Twitter at polling the United States 2016 presidential election. In 2017 IEEE 3rd International Conference on Collaboration and Internet Computing (CIC). IEEE, 283-290.

[39] Eitan D Hersh. 2015. Hacking the electorate: How campaigns perceive voters. Cambridge University Press.

[40] D Sunshine Hillygus and Todd G Shields. 2008. The persuadable voter: Wedge issues in presidential campaigns. Princeton University Press.

[41] Paul Hitlin and Lee Rainie. 2019. Facebook Algorithms and Personal Data. Pew Research Center: Internet \& Technology (2019). https://www.pewinternet.org/2019/01/16/facebook-algorithms-and-personaldata/.

[42] Donna L Hoffman and Marek Fodor. 2010. Can you measure the ROI of your social media marketing? MIT Sloan Management Review 52, 1 (2010), 41.

[43] Roope Jaakonmäki, Oliver Müller, and Jan Vom Brocke. 2017. The impact of content, context, and creator on user engagement in social media marketing. In Proceedings of the 50th Hawaii international conference on system sciences. AIS.

[44] Young Mie Kim, Jordan Hsu, David Neiman, Colin Kou, Levi Bankston, Soo Yun Kim, Richard Heinrich, Robyn Baragwanath, and Garvesh Raskutti. 2018. The stealth media? Groups and targets behind divisive issue campaigns on Facebook. Political Communication 35, 4 (2018), 515-541.

[45] Simon Kingsnorth. 2019. Digital marketing strategy: An integrated approach to online marketing. Kogan Page Publishers.

[46] Ralf Kneuper and Mark Perlitz. 2019. Datenschutz bei nutzungsbasierter OnlineWerbung. Datenschutz und Datensicherheit 43, 8 (2019), 502-506.

[47] Aleksandra Korolova. 2010. Privacy Violations Using Microtargeted Ads: A Case Study. In ICDM Workshops. IEEE Computer Society, 474-482.

[48] Daniel Kreiss. 2016. Prototype politics: Technology-intensive campaigning and the data of democracy. Oxford University Press.

[49] Daniel Kreiss and Shannon C. Mcgregor. 2019. The "Arbiters of What Our Voters See": Facebook and Google's Struggle with Policy, Process, and Enforcement around Political Advertising. Political Communication (2019), 1-24.

[50] Dokyun Lee, Kartik Hosanagar, and Harikesh S Nair. 2018. Advertising content and consumer engagement on social media: Evidence from Facebook. Management Science 64, 11 (2018), 5105-5131.

[51] Lynda Lee Kaid, Juliana Fernandes, and David Painter. 2011. Effects of political advertising in the 2008 presidential campaign. American Behavioral Scientist 55, 4 (2011), 437-456.

[52] Janette Lehmann, Mounia Lalmas, Elad Yom-Tov, and Georges Dupret. 2012. Models of user engagement. In International conference on user modeling, adaptation, and personalization. Springer, 164-175.

[53] Vishal Monga and Brian L Evans. 2006. Perceptual image hashing via feature points: performance evaluation and tradeoffs. IEEE transactions on Image Processing 15, 11 (2006), 3452-3465.

[54] Matthew P Motta and Erika Franklin Fowler. 2016. The content and effect of political advertising in US campaigns. In Oxford Research Encyclopedia of Politics.

[55] Mozilla. 2019. Facebook's Ad Archive API is Inadequate. The Mozilla Blog (2019). https://blog.mozilla.org/blog/2019/ 04/29/facebooks-ad-archive-api-isinadequate/.

[56] Nanigans. 2019. Global Facebook Advertising Benchmark Report Q1 2019. (2019).

[57] David W Nickerson and Todd Rogers. 2014. Political campaigns and big data. Journal of Economic Perspectives 28, 2 (2014), 51-74.

[58] Robert W Palmatier and Kelly D Martin. 2019. Big Data's Marketing Applications and Customer Privacy. In The Intelligent Marketer's Guide to Data Privacy. Springer, 73-92.

[59] Orestis Papakyriakopoulos, Simon Hegelich, Morteza Shahrezaye, and Juan Carlos Medina Serrano. 2018. Social media and microtargeting: Political data processing and the consequences for Germany. Big Data \& Society 5, 2 (2018).

[60] Flora Poecze, Claus Ebster, and Christine Strauss. 2018. Social media metrics and sentiment analysis to evaluate the effectiveness of social media posts. Procedia computer science 130 (2018), 660-666.

[61] Gustavo Resende, Philipe Melo, Hugo Sousa, Johnnatan Messias, Marisa Vasconcelos, Jussara Almeida, and Fabrício Benevenuto. 2019. (Mis) Information Dissemination in WhatsApp: Gathering, Analyzing and Countermeasures. In The World Wide Web Conference 2019. ACM, 818-828.

[62] Filipe N. Ribeiro, Koustuv Saha, Mahmoudreza Babaei, Lucas Henrique, Johnnatan Messias, Fabricio Benevenuto, Oana Goga, Krishna P. Gummadi, and Elissa M. Redmiles. 2019. On Microtargeting Socially Divisive Ads: A Case Study of RussiaLinked Ad Campaigns on Facebook. In Proceedings of the Conference on Fairness, Accountability, and Transparency (FAT '19). ACM, 140-149.

[63] Juan Carlos Medina Serrano, Morteza Shahrezaye, Orestis Papakyriakopoulos, and Simon Hegelich. 2019. The Rise of Germany's AfD: A Social Media Analysis. In Proceedings of the 10th International Conference on Social Media and Society (SMSociety '19). ACM, 214-223. 
[64] Morteza Shahrezaye, Orestis Papakyriakopoulos, Juan Carlos Medina Serrano, and Simon Hegelich. 2019. Measuring the Ease of Communication in Bipartite Social Endorsement Networks: A Proxy to Study the Dynamics of Political Polarization. In Proceedings of the 10th International Conference on Social Media and Society (SMSociety '19). ACM, 158-165.

[65] Till Speicher, Muhammad Ali, Giridhari Venkatadri, Filipe Nunes Ribeiro, George Arvanitakis, Fabrício Benevenuto, Krishna P. Gummadi, Patrick Loiseau, and Alan Mislove. 2018. Potential for Discrimination in Online Targeted Advertising. In FAT (Proceedings of Machine Learning Research), Vol. 81. PMLR, 5-19.

[66] Emil Stjernholm. 2014. Political ads in the Swedish 2006 and 2010 parliamentary elections: Focus on the Sweden Democrats and the role of audiovisual media. Online fournal of Communication and Media Technologies 4, 2 (2014), 32-50.

[67] SZ. 2019. So hat Deutschland gewählt. Süddeutsche Zeitung (2019). Retrieved September 06, 2019 from https://www.sueddeutsche.de/politik/europawahlendeutschland-ergebnisse-1.4456025.

[68] Monika Taddicken. 2014. The 'privacy paradox' in the social web: The impact of privacy concerns, individual characteristics, and the perceived social relevance on different forms of self-disclosure. Fournal of Computer-Mediated Communication 19,2 (2014), 248-273.
[69] Zeynep Tufekci. 2015. Algorithmic harms beyond Facebook and Google: Emergent challenges of computational agency. Colo. Tech. Lf 13 (2015), 203.

[70] Michail Vafeiadis, Ruobing Li, and Fuyuan Shen. 2018. Narratives in political advertising: An analysis of the political advertisements in the 2014 midterm elections. Fournal of Broadcasting \& Electronic Media 62, 2 (2018), 354-370.

[71] Giridhari Venkatadri, Elena Lucherini, Piotr Sapiezynski, and Alan Mislove. 2019. Investigating sources of PII used in Facebook's targeted advertising. PoPETs 2019, 1 (2019), 227-244.

[72] Julia Carrie Wong. 2019. Document reveals how Facebook downplayed early Cambridge Analytica concerns. The Guardian. Retrieved September 06, 2019 from https://www.theguardian.com/technology/2019/aug/23/cambridgeanalytica-facebook-response-internal-document.

[73] Jing Zhang and En Mao. 2016. From online motivations to ad clicks and to behavioral intentions: An empirical study of consumer response to social media advertising. Psychology \& Marketing 33, 3 (2016), 155-164.

[74] Frederik Zuiderveen Borgesius, Judith Möller, Sanne Kruikemeier, Ronan Ó Fathaigh, Kristina Irion, Tom Dobber, Balazs Bodo, and Claes H de Vreese. 2018. Online political microtargeting: Promises and threats for democracy. Utrecht Law Review 14, 1 (2018), 82-96. 